\title{
Cosmic rays and high energy emission from starburst galaxies
}

\author{
Brian C. Lacki ${ }^{1}$ and Todd A. Thompson ${ }^{2}$ \\ ${ }^{1}$ Jansky Fellow \& Institute for Advanced Study; Einstein Drive, Princeton, NJ 08540, USA \\ ${ }^{2}$ Department of Astronomy and Center for Cosmology and AstroParticle Physics, Ohio State \\ University; 140 W. 18th Ave, Columbus, OH 43210, USA \\ email: brianlacki@ias.edu
}

\begin{abstract}
The nearby starburst galaxies M82 and NGC 253 are now detected in GeV and $\mathrm{TeV} \gamma$-rays, allowing us to directly study cosmic rays (CRs) in starburst galaxies. Combined with radio observations, the detections constrain the propagation and density of CRs in these starbursts. We discuss the implications for "proton calorimetry", whether CR protons cool through pion losses before escaping these galaxies. The ratio of $\gamma$-ray and radio luminosities constrains how much of the CR electron cooling is due to synchrotron losses. As for leptonic emission, we predict that synchrotron and Inverse Compton emission make up $\sim 1-10 \%$ of the unresolved hard X-ray emission from M82, and a few percent or less of the total X-ray emission from starbursts. A detection of these components would inform us of the magnetic field strength and $10-100 \mathrm{TeV}$ electron spectrum. We conclude by discussing the prospects for detecting leptonic $\mathrm{MeV} \gamma$-rays from starbursts and the cosmic $\gamma$-ray background.
\end{abstract}

Keywords. galaxies: starburst, gamma rays: theory, gamma rays: observations, X-rays: galaxies

As the conference theme says, galaxies are detected over more than 18 orders of magnitude in energy. This is not just true for the Milky Way and active galactic nuclei anymore, but now for starburst galaxies as well: M82 is detected from $38 \mathrm{MHz}(0.2 \mu \mathrm{eV}$; Kellermann, Pauliny-Toth, \& Williams 1969) to $4 \mathrm{TeV}$ (970 YHz; Acciari et al. 2009). Thermal emission lies in the middle of the spectrum (millimeter to X-rays), but the rest is nonthermal. Nonthermal emission comes mainly from cosmic rays (CRs), high energy particles accelerated by supernova remnant shocks or other star formation processes. Protons (and other nuclei) make up most of the energy density in CRs above a GeV, but CR electrons and positrons $\left(e^{ \pm}\right)$are also present. Starbursts have much star formation in small volumes, resulting in large CR energy densities.

CRs radiate in a variety of ways. These processes have different spectra, peaking at different energies. Radiation can be leptonic, from $\mathrm{CR} e^{ \pm}$, or hadronic, from CR protons. Synchrotron radiation is emitted by $e^{ \pm}$gyrating in magnetic fields and is a very broad continuum (with a characteristic frequency $\nu_{\text {synch }} \propto B E_{e}^{2}$, where $E_{e}$ is the electron energy and $B$ is the magnetic field strength) spanning from radio to $\mathrm{X}$-rays. Inverse Compton (IC) is emitted by $e^{ \pm}$upscattering individual photons; it too is a very broad continuum (characteristic IC photon energy $E_{\mathrm{IC}} \propto E_{e}^{2} \varepsilon$, where $\varepsilon$ is the energy of a typical low energy photon), stretching from X-rays to $\gamma$-rays. Bremsstrahlung is emitted by $e^{ \pm}$ being deflected by the electric fields of ambient atoms and therefore correlates with gas density; it is a narrow continuum feature $\left(E_{\mathrm{brems}} \propto E_{e}\right)$ that peaks at a few hundred $\mathrm{MeV}$. Finally, CR protons can inelastically collide with ambient gas atoms to produce pions, uncharged $\left(\pi^{0}\right) 1 / 3$ of the time or charged $\left(\pi^{+}\right.$or $\left.\pi^{-}\right) 2 / 3$ of the time. Neutral pions quickly decay into pionic $\gamma$-rays; because of the kinematics of the pion production process, the pionic $\gamma$-rays form a plateau $\left(E_{\text {pionic }} \propto E_{p}\right)$ reaching up to $\gtrsim 100 \mathrm{TeV}$, but 
dropping off quickly below $\sim 70 \mathrm{MeV}$. If we had the full broadband spectra of starburst galaxies we would learn both about their CRs and the environments they travel in.

The GeV-TeV Band: CR Protons at Last. Although protons make up the bulk of the CR energy density in star-forming galaxies, only the recent detections of starbursts by Fermi (Abdo et al. 2010), HESS (Acero et al. 2009), and VERITAS (Acciari et al. 2009) gave us direct evidence for them. Previously, their presence was merely inferred from equiparition arguments on radio observations (e.g., Akyuz, Brouillet, \& Ozel 1991). The $\gamma$-ray detections indicate $U_{\mathrm{CR}} \approx 100-300 \mathrm{eV} \mathrm{cm}^{-3}$ within M82 and NGC 253's starbursts (Acero et al. 2009; Lacki et al. 2011).

Unlike the Milky Way, starbursts may be "proton calorimeters", in which most of the energy in CR protons goes into making pions, since they have high gas densities. In this limit, $1 / 3$ of the injected $\mathrm{CR}$ proton power $\left(L_{\mathrm{CR}}\right)$ is converted into pionic $\gamma$-rays; the rest goes into secondary $e^{ \pm}$and neutrinos. The $\gamma$-ray detections allow us to estimate the fraction $F_{\text {cal }}$ of $\mathrm{CR}$ power that ends up in pionic products. We have some idea of the power being pumped into CRs in M82 and NGC 253: it should scale with the supernova (or massive star formation) rate. From their IR luminosities, we estimate supernova rates of $0.06 \mathrm{yr}^{-1}$ in M82 and $0.02 \mathrm{yr}^{-1}$ in NGC 253's core (though with large uncertainties 0.1 to $0.3 \mathrm{yr}^{-1}$ are sometimes quoted). Assuming that each supernova injects $10^{50} \mathrm{ergs}$ of CR protons, we find that $F_{\text {cal }}=3 L_{\gamma} / L_{\mathrm{CR}} \approx 40 \%$ of the CR power in M82 and NGC 253 goes into pionic losses (Lacki et al. 2011). By contrast, in the Milky Way only a few percent of the CR proton power ends up in pions (e.g., Strong et al. 2010).

Pionic $\gamma$-rays necessarily come with pionic $e^{ \pm}$: if these cooled only by synchrotron emission, the Fermi detections imply that M82 and NGC 253 should be very bright in $\mathrm{GHz}$ radio. Since half as much power in $\gamma$-rays goes into secondary $e^{ \pm}$as pionic $\gamma$-rays, and synchrotron radiation spreads each dex in $e^{ \pm}$energy into two dex of synchrotron frequency, we expect that $\nu L_{\nu}(\mathrm{GeV}) f_{\text {synch }} \beta=4 \nu L_{\nu}(\mathrm{GHz})$, where $\beta \approx 1$ corrects for differences in observed $e^{ \pm}$energy and $f_{\text {synch }}$ is the fraction of $e^{ \pm}$power going into synchrotron. In fact, M82 and NGC 253 are much less luminous in radio than in GeV $\gamma$-rays, implying that $f_{\text {synch }} \approx 0.1$. This indicates the importance of non-synchrotron energy losses - IC, bremsstrahlung, ionization, or escape - for these $e^{ \pm}$(Lacki et al. 2011).

The keV Band: Crucial Information Buried by Foregrounds. Several decades ago, it was thought that starbursts would be bright sources of IC X-rays, because they have intense infrared radiation fields and a supply of CR $e^{ \pm}$evident from their radio emission (e.g., Hargrave 1974). Since $e^{ \pm}$of similar energies emit $\mathrm{GHz}$ radio synchrotron and $\mathrm{keV}$ IC, a ratio of the power in these two bands could tell us directly about the magnetic field strength in starbursts. However, a variety of arguments indicate starburst galaxies have strong magnetic fields (e.g., Völk 1989; Thompson et al. 2006; Robishaw, Quataert, \& Heiles 2008; Persic et al. 2010; Crocker et al. 2010), meaning that the ratio of radio and IC emission should be large. Furthermore, the amount of $\mathrm{GeV}$ emission observed limits the IC emission since the IC continuum should stretch from X-rays to TeV energies.

But another source of nonthermal X-ray emission in starbursts is synchrotron emission from $10-100 \mathrm{TeV} e^{ \pm}$. The strong magnetic fields in starbursts means that lower energy $e^{ \pm}$can contribute to synchrotron than in normal galaxies (c.f. Protheroe \& Wolfendale 1980, Aharonian \& Atoyan 2000). These $e^{ \pm}$can include primaries, pionic secondaries, or pair $e^{ \pm}$produced by far-infrared photons annihilating $10-100 \mathrm{TeV}$ photons through the $\gamma+\gamma \rightarrow e^{+}+e^{-}$process (e.g., Aharonian, Atoyan \& Nagapetyan 1983; Torres 2004). The synchrotron and IC contributions have different spectra: IC emission should be hard $(\Gamma \approx 1-1.5)$ whereas synchrotron emission should be soft $(\Gamma \approx 2)$.

Using one-zone models to solve the leaky box equation for CR populations (c.f. Torres 2004; Lacki, Thompson, \& Quataert 2010), we have calculated the nonthermal leptonic 
X-ray emission from several nearby starbursts (Lacki \& Thompson 2010b). After applying constraints from the observed radio and $\gamma$-ray emission, we find that only a few percent of the diffuse, unresolved X-ray emission from these starbursts is synchrotron or IC. If the gas density is low, the synchrotron X-ray contribution depends strongly on where the primary electron spectrum (assumed to be a single power law) cuts off, since there are few secondaries or pair $e^{ \pm}$at $10-100 \mathrm{TeV}$. In M82, synchrotron and IC account for only $\lesssim 10 \%$ of the diffuse, unresolved X-ray emission, most of which probably comes from hot X-ray gas (Strickland \& Heckman 2007). The diffuse X-ray emission in turn is only a minority of the total X-ray emission, which largely is expected to come from X-ray binaries (e.g., Persic et al. 2004). In Arp 220, synchrotron may be about a quarter of the observed 2-10 keV X-rays if it is not absorbed by hydrogen, but hydrogen absorption from the huge gas column densities likely reduces that fraction. In general we find that synchrotron and IC are no more than $\sim 2-3 \%$ of the total $2-10 \mathrm{keV}$ luminosity of starbursts.

The MeV Band: The Hole in our Rainbow. GeV and TeV observations tell us about protons but only indirectly connect with $\mathrm{GHz}$-emitting $e^{ \pm}$, while $\mathrm{keV}$ observations must contend with large foregrounds. In between those energies, in the $\mathrm{MeV}$ band, leptonic emission should be the primary source of luminosity (Fig. 1).

Detailed models of CR populations in M82 and NGC 253 predict IC and bremsstrahlung emission at $\mathrm{MeV}$ energies (e.g., de Cea del Pozo et al. 2009). Unfortunately, it is dimmer than the pionic emission, since there are more $\geqslant \mathrm{GeV}$ protons than $e^{ \pm}$. An even bigger problem is that current instruments' sensitivity to $\mathrm{MeV} \gamma$-rays are far behind that of Fermi-LAT at $30 \mathrm{MeV}$ and above. These sensitivities must improve by a factor of $a$ thousand to detect M82 and NGC 253, the brightest starbursts in the sky.

Could we instead detect all the starbursts in the Universe in the form of the diffuse $\gamma$-ray background (c.f. Pavlidou \& Fields 2002, Thompson, Quataert, \& Waxman 2007)?

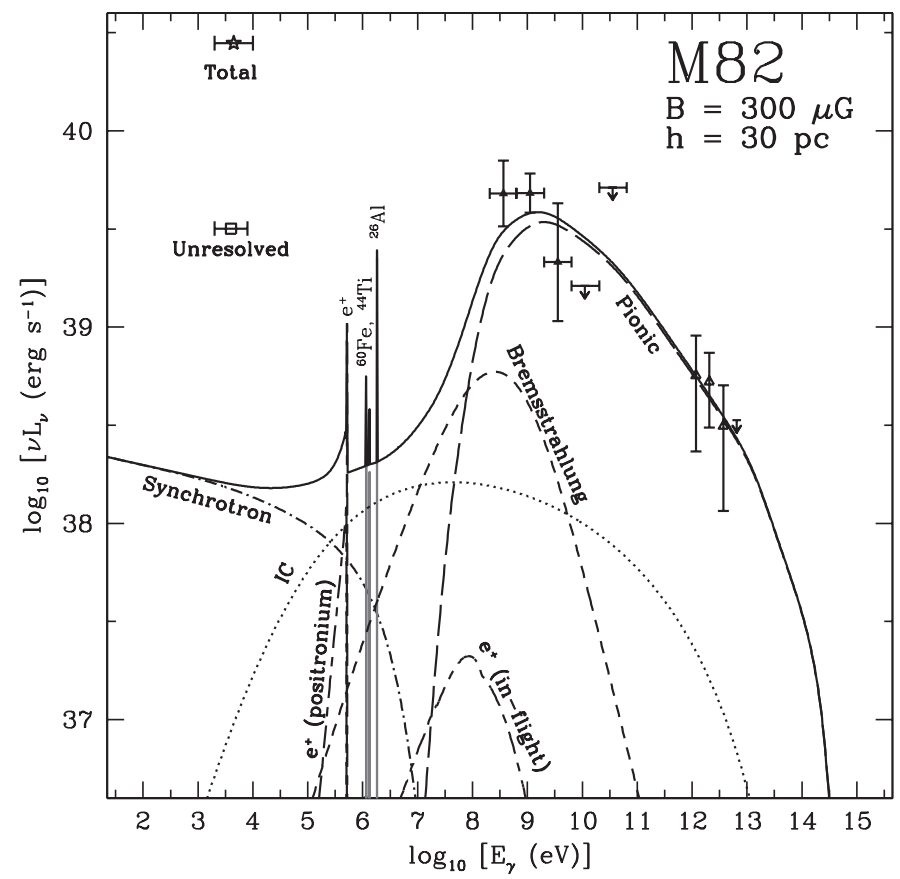

Figure 1. Predicted high energy spectrum of M82 (ignoring X-ray absorption). Data points are Cappi et al. 1999, Strickland \& Heckman 2007, Abdo et al. 2010, and Acciari et al. 2009. 
By using the radiation spectra from one-zone models of the Milky Way, M82, and NGC 253 as templates for star-formation throughout the Universe, we recently calculated the expected $\gamma$-ray background at $\mathrm{MeV}$ and $\mathrm{GeV}$ energies. We find that starbursts have much absolute power at $\mathrm{GeV}$ energies, but normal galaxies have higher $\mathrm{MeV}$ to $\mathrm{GeV}$ ratios. In our fiducial model, starbursts are more important above a few $\mathrm{GeV}$, normal galaxies below that, together making up $\sim 1 / 3$ of the $\mathrm{GeV} \gamma$-ray background and a few percent of the MeV background (Lacki et al. 2011, in prep.).

In high- $z$ normal galaxies, IC losses off the CMB rapidly dominate the lifetimes of $\mathrm{CR} e^{ \pm}$because $t_{\mathrm{IC}}^{\mathrm{CMB}} \propto U_{\mathrm{CMB}}^{-1} \propto(1+z)^{-4}$. Thus power that would normally go into other energy loss processes (including radio emission) or escape instead gets channeled into the $\mathrm{MeV}$-scale IC emission. Since protons escape easily in normal galaxies, the leptonic contribution can fill in the "pion bump". In starburst galaxies, the IC losses from the CMB are insignificant compared to all the other strong losses in the extreme environments (the "buffering" of Lacki \& Thompson 2010a). Furthermore, in the more proton calorimetric starbursts, the pionic emission is more dominant.

\section{Acknowledgements}

BCL is supported by a Jansky Fellowship from the National Radio Astronomy Observatory. NRAO is operated by Associated Universities, Inc., under cooperative agreement with the National Science Foundation. BCL also acknowledges discussions and collobration with John Beacom and Shunsaku Horiuchi on the forthcoming paper on the MeV background. TAT is supported in part by an Alfred P. Sloan Foundation Fellowship and by NSF grant AST-0908816.

\section{References}

Abdo, A. A., Ackermann, M., Ajello, M., et al. 2010, ApJL, 709, L152

Acciari, V. A., Aliu, E., Arlen, T., et al. 2009, Nature, 462, 770

Acero, F., Aharonian, F., Akhperjanian, A. G., et al. 2009, Science, 326, 1080

Aharonian, F. A., Atoyan, A. M., \& Nagapetyan, A. M. 1983, Astrophysics, 19, 187

Aharonian, F. A. \& Atoyan, A. M. 2000, A\& $A, 362,937$

Akyuz, A., Brouillet, N., \& Ozel, M. E. 1991, A\&A, 248, 419

Cappi, M., Persic, M., Bassani, L., et al. 1999, A\& $A, 350,777$

Crocker, R. M., Jones, D. I., Melia, F., Ott, J., \& Protheroe, R. J. 2010, Nature, 463, 65

de Cea del Pozo, E., Torres, D. F., Rodriguez, A. Y., \& Reimer, O. 2009, arXiv:0912.3497

Hargrave, P. J. 1974, MNRAS, 168, 491

Kellermann, K. I., Pauliny-Toth, I. I. K., \& Williams, P. J. S. 1969, ApJ, 157, 1

Lacki, B. C., Thompson, T. A., \& Quataert, E. 2010, ApJ, 717, 1

Lacki, B. C. \& Thompson, T. A. 2010a, ApJ, 717, 196

Lacki, B. C. \& Thompson, T. A. 2010b, arXiv:1010.3030

Lacki, B. C., Thompson, T. A., Quataert, E., Loeb, A., \& Waxman, E. 2011, ApJ, 734, 107

Pavlidou, V. \& Fields, B. D. 2002, ApJL, 575, L5

Persic, M., Rephaeli, Y., Braito, V., et al. 2004, A\&A, 419, 849

Persic, M. \& Rephaeli, Y. 2010, MNRAS, 403, 1569

Protheroe, R. J. \& Wolfendale, A. W. 1980, A\&A, 92, 175

Robishaw, T., Quataert, E., \& Heiles, C. 2008, ApJ, 680, 981

Strickland, D. K. \& Heckman, T. M. 2007, ApJ, 658, 258

Strong, A. W., Porter, T. A., Digel, S. W., et al. 2010, ApJL, 722, L58

Thompson, T. A., et al. 2006, ApJ, 645, 186

Thompson, T. A., Quataert, E., \& Waxman, E. 2007, ApJ, 654, 219

Torres, D. F. 2004, ApJ, 617, 966

Völk, H. J. 1989, $A \mathscr{E} A, 218,67$ 